Notfall Rettungsmed 2007 · 10:165-166 DOI 10.1007/s10049-007-0915-6

Online publiziert: 21. April 2007

๑) Springer Medizin Verlag 2007
S. Reiter-Theil ${ }^{1} \cdot$ W. Dick ${ }^{2}$

${ }^{1}$ Institut für Angewandte Ethik und Medizinethik, Basel

2 Klinik für Änästhesiologie, Johannes Gutenberg-Universität Mainz

\title{
Ethik in der Notfallmedizin
}

Zu keiner Zeit waren die Konflikte zwischen den Möglichkeiten der medizinischen Versorgung des Notfallpatienten auf der einen Seite und den finanziellen Ressourcen auf der anderen ausgeprägter als heute. Damit haben auch die ethischen Kontroversen gegenüber bisherigen Problemanalysen noch deutlich an Brisanz zugenommen [1, 2]. Es geht heute nicht mehr vorrangig darum, die klinisch Tätigen davon zu überzeugen, dass die Selbstbestimmung des Patienten zu respektieren und zu fördern ist. Diesist für viele bereits integraler Bestandteil ihrer klinischen Alltagsethik. Das Entwicklungspotenzial und die Expansion der Medizin selbst öffnet die Schere zwischen dem technisch Möglichen (und dann auch Erwarteten) und dem, was unsere Ressourcen hergeben, immer weiter. Der vorliegende Themenschwerpunkt "Ethik" greift diese Problematik auf und stellt dazu aktuelle Aufsätze vor, die auf der Grundlage eines Expertenkolloquiums im November 2004 in Inzlingen bei Basel erarbeitet worden sind, welches durch die Förderung der Stiftung BINZ (Lorch) ermöglicht wurde.

Der Notfallpatient gilt bis heute als ,sakrosankt" mit der Konsequenz, dass grundsätzlich alles Erdenkliche für ihn zu tun ist - zumindest bis zu seiner Aufnahme in die Klinik. Kaum jemand wagt es, irgendeine Maßnahme zu unterlassen oder eine niedrigere Versorgungskategorie (diese sind ohnehin limitiert) auszuwählen, nur unter dem Aspekt, Geld einsparen zu wollen. Dabei bleibt andererseits oft die Humanität gegenüber dem Notfallpatienten auf der Strecke. Ungeachtet seines Alters, seiner Lebens- prognose - z. B. auf der Basis einer konsumierenden Erkrankung - wird die Frage nach dem Sinn der Maßnahmen häufig au$\beta e r$ Acht gelassen. So wird oft genug ein Behandlungsergebnis erzielt, das in Form einer bleibenden Hirnschädigung, eines permanenten Komas oder einer Verschlimmerung des Leidens imponiert. Daraus resultiert die Spannung, zu viel oder zu wenig für den Patienten in der Notfallsituation bzw. für seine Rettung getan zu haben. Hinzu kommen Befürchtungen vor rechtlichen Konsequenzen.

\section{จ Humanität bleibt gegenüber dem Notfallpatienten oft auf der Strecke}

Ein alltägliches Beispiel: Wird auf der einen Seite etwa eine Reanimation im Krankenhaus mit dem Resultat eines apallischen Ausgangs bei einem Patienten mittleren Alters zunächst als insuffiziente Reanimation inkriminiert, so wird im Umkehrschluss im gleichen Falle sekundär argumentiert, man hätte diese Reanimation angesichts der möglicherweise langen Latenz zwischen Eintritt des HerzKreislauf-Stillstands und Erstmaßnahmen überhaupt nicht beginnen dürfen. In diese Diskussion flossen auch schon zusätzlich parteipolitische Aspekte - insbesondere von jüngeren Politikern - ein, die das Recht auf eine ergebnisorientierte Therapie von Altersobergrenzen abhängig machen wollten, wie das in einigen Ländern (innerhalb und außerhalb Europas) bereits praktiziert wird. Dies wurde zum Beispiel am Polytrauma festgemacht, mit der Begründung, dass die Erfolgsaussichten nach einem Herz-Kreislauf-Stillstand im Gefolge eines Polytraumas extrem gering seien und daher kaum eine Indikation zur Reanimation bestünde, ungeachtet der Tat- sache, dass polytraumatisierte Patienten häufig noch relativ jung sind.

Wie das Expertenkolloquium, so setzt auch das vorliegende Heft an der Schnittstelle zwischen Rettungsmedizin und Notaufnahme an und versucht, den Dialog zwischen den beteiligten Disziplinen zu vertiefen. Im vorliegenden Heft beschreiben R. Bingisser, O. Gratzl und D. Scheidegger die vielfältigen Probleme an der Schnittstelle zwischen Rettungsdienst und Klinik in der Notaufnahme (Potenzial für Irrtümer, Inkompetenzen und Inkongruenzen). Darauf folgt die Untersuchung zu Problemen der Mittelverteilung im Gesundheitswesen aus der Sicht des Managements (P. Berchtold) und der Ethik (F. J. Illhardt). Dabei postuliert Berchtold die Notwendigkeit von Lösungen durch Vernetzung und Kooperation. In seinem medizinökonomischen Beitrag geht er auch davon aus, es gebe keine gesicherte Evidenz für das Vorliegen von (impliziter) Rationierung, jedenfalls nicht für die Schweiz - eine Position, die nicht unwidersprochen geblieben ist. Mit der Frage der präklinischen Wiederbelebung im hohen Alter befasst sich der Beitrag von M. Mohr aus medizinischer Sicht und arbeitet heraus, dass eine Benachteiligung von alten Patienten nicht gerechtfertigt sei. Der Beitrag nimmt auch zu der Möglichkeit Stellung, ob und wie Patientenverfügungen in der Notfallmedizin berücksichtigt werden können - eine Frage, die im deutschsprachigen Raum bisher noch zu wenig Eingang in die Praxis gefunden hat.

\section{> Der Dialog zwischen den beteiligten Disziplinen muss vertieft werden}

Studien zu ethischen Fragen in der Medizin bedienen sich heute zunehmend auch empirischer Methoden, um ihren Gegenstand 
adäquat zu erfassen, bevor dieser einer Bewertung unterzogen wird. Dies veranschaulicht der Beitrag von S. Reiter-Theil und H. Albisser Schleger, in dem aufder Grundlage von 3 Studien (zur Notfallmedizin, Intensivmedizin und Geriatrie sowie Allgemein- bzw. Innere Medizin) gezeigt wird, dass das Alter faktisch durchaus Einfluss auf Therapieentscheidungen hat und dass die implizite oder verdeckte Rationierung bereits Realität ist. Insbesondere wird anhand aktueller Daten aus 4 europäischen Ländern gezeigt, dass hohes Alter ein Risiko für das Vorenthalten von Maßnahmen mit sich bringt und dass erhöhte Wachsamkeit für diese Fragen angezeigt ist.

Dernächste Themenblock widmet sich der Frage der Laienreanimation. J. Bahr liefert hierzu grundlegende Informationen zur Lage in Deutschland. Rechtliche(V. Dittmann) und psychologische Aspekte der Laienhilfe ergänzen die Darstellung dieses wichtigen Bereichs. B. Gasch berichtet aus eigener großer Erfahrung, welche psychologischen und ethischen Herausforderungen mit der Laienhilfe verbunden sind.

Neben der Problemanalyse soll dieses Schwerpunktheft auch die Qualifizierung der im Rettungsdienst Tätigen unterstützen. So diskutieren K. Lindner, W. Ummenhofer und S. Reiter-Theil, welche Bedürfnisse und Probleme das Rettungspersonal (Sanitäter und Ärzte) selbst in Bezug auf die Bewältigung der schwierigen ethischen Anforderungen artikuliert. Sie stützen sich auf Daten einer repräsentativen Interviewstudie in der "Sanität Basel" und formulieren Empfehlungen für die ethische Fortbildung. Dieser Ansatz wird noch erweitert durch den Beitrag von W. Ummenhofer und M. Zürcher über die medizinische Ausbildung von Rettungspersonal.

Wir hoffen, mit dem Leitthema „Zwischen Notfallort und Notaufnahme: ethische Entscheidungen über lebenserhaltende Maßnahmen" einen Beitrag zu einer Diskussion zu leisten. Diese Diskussion ist angesichts der immer prekärer werdenden finanziellen Situation der Medizin und ihrer immer weiter steigenden technischen und medikamentösen Möglichkeiten unvermeidbar geworden. Folgende Fragen müssen gestellt und beantwortet werden:

1. Wenn jeder Notfallpatient bereits in der Prähospitalphase, aber auch in der Notaufnahme und erst recht in der Spezial- klinik, die jeweils optimale Behandlung erfahren soll (wie dies die Politik immer wieder verlangt), müssen dann nicht die Beiträge der künftigen Patienten bzw. der Allgemeinheit notwendigerweise den finanziellen Erfordernissen angepasst, $d$.h. erhöht werden?

2. Daraus folgend: Sind wir bereit, mehr für unsere Gesundheit auszugeben?

3. Wenn diese Forderung nach einer Erhöhung des Budgets - insbesondere von Seiten der Politik - immer wieder abgelehnt wird, muss dann nicht die Politik auch die „ethischen Rahmenbedingungen"vorgeben und Kriterien benennen, nach denen eine Rationierung medizinischer Leistungen vorgenommen werden soll?

4. Konsequenterweise muss die Politik dann aber auch die Frage beantworten, welche Ressourcen welchen Bevölkerungskreisen zur Verfügung gestellt werden sollen, vom extrem Frühgeborenen bis zum extrem alten Patienten.

Über Rationierung (die im Übrigen längst existiert, auch wenn sie oft bestritten wird) sprechen viele nur hinter vorgehaltener Hand. Die Medizin selbst kann allein allenfalls durch standardisierte Behandlungsabläufe und Inhalte festlegen, was eine ,ausreichende" Therapie bedeutet und welchen Effekt sie hat. Jedes Abweichen von diesem Standard stell teine Verletzung von Konventionen dar, die - wenn überhaupt - nur mit guten Gründen medizinisch und ethisch gerechtfertigt werden kann. Solche guten Gründe bedürfen einer transparenten Diskussion und Konsensbildung auf breiter Basis. Diese Diskussion muss anhaltend gepflegt werden, damit die Argumente und Kriterien stetig weiterentwickelt und angepasst werden können. In diesem Spannungsfeld gibt es noch viel zu tun, und die Probleme werden sich nur interdisziplinär lösen lassen.

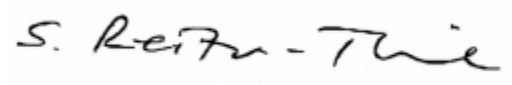

Prof. Dr. Stella Reiter-Theil

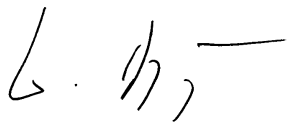

Prof. Dr. Dr. h.c. Wolfgang Dick

\section{Korrespondenzadresse \\ Prof. Dr. S. Reiter-Theil}

Institut für Angewandte Ethik und Medizinethik Missionsstrasse 21 A, 4003 Basel, Schweiz s.reiter-theil@unibas.ch

Danksagung. Wir danken der Stiftung BINZ, Lorch, sehr herzlich für die großzügige Förderung des Expertenkolloquiums, welches das Zustandekommen der Arbeiten dieses Schwerpunktheftes möglich gemacht hat.

\section{Literatur}

1. Hick C, Bengel J, Mohr M, Reiter-Theil S (Hrsg) (2000) Ethik in der präklinischen Notfallmedizin. Übersicht zu ethischen Aspekten im Rettungsdienst und in der Notfallmedizin. Schriftenreihe zum Rettungswesen, DRK-Landesverband Westfalen-Lippe, Nottuln

2. Hick C, Bengel J, Mohr M, Reiter-Theil S (2003) Ethische Aspekte der präklinischen Notfallversorgung. Notfall Rettungsmed 6: 115-121 